\title{
In Situ Investigation of Calcium Oxalate Mineralization
}

David J. Banner ${ }^{1}$, Emre Firlar ${ }^{1,2}$, Kun $\mathrm{He}^{2}$, Jodi Finlay ${ }^{1}$, Reza Shahbazian Yassar $^{2}$ and Tolou Shokuhfar ${ }^{1,2}$

1. University of Illinois at Chicago, Department of Bioengineering, Chicago, IL.

2. University of Illinois at Chicago, Department of Mechanical and Industrial Engineering, Chicago, IL.

The importance of nanoscale crystallization phenomena of calcium salts on their observed macroscale biomineralizations have been recognized in recent years [1]. Calcium oxalate ( $\mathrm{CaOx})$, a calcium salt, is the major pathological biomineralization in human kidney stones, which affect hundreds of millions of people worldwide and cost over $\$ 5$ billion dollars per year [2]. Kidney stones constitute a large volume of hospital admissions and over 1.3 million annual emergency department visits [2]. These high admission rates place a heavy burden on clinical staff and on healthcare systems worldwide. Due to the substantial human and economic burden, further research and imaging of $\mathrm{CaOx}$ nanoscale biomineralization is imperative. However, the electron microscopy (EM) necessary to visualize nanoscale structures of $\mathrm{CaOx}$ is technically difficult due to the beam sensitivity of the mineral, the effect of the vacuum, and the sample preparation. Furthermore, in situ nanoscale formation of beam sensitive $\mathrm{CaOx}$ has been limited to twodimensional techniques such as atomic force microscopy. In situ EM enables real-time imaging of nanoscale mineralization phenomena as well as chemical and structural characterization techniques without requiring preservation of the sample. An improved understanding of classical and non-classical mineralization may yield insight into high efficacy $\mathrm{CaOx}$ treatments and preventative medicine.

Here, $\mathrm{CaOx}$ was synthesized within an EM liquid flow holder (LF) (Figure 1). Calcium chloride $\left(\mathrm{CaCl}_{2}\right)$ was placed upon the lower $\mathrm{SiN}$ window (Figure 1A). The upper window was placed over the $\mathrm{CaCl}_{2}$, encapsulating the $\mathrm{CaCl}_{2}$ sample within the LF. Sodium oxalate $(\mathrm{NaOx})$ was then slowly flowed through LF enclosure and imaged via Scanning Transmission EM (STEM) (Figure 1B). CaOx formed within the LF via chemical reaction: $\mathrm{Na}_{2} \mathrm{C}_{2} \mathrm{O}_{4}+\mathrm{CaCl}_{2} \rightarrow \mathrm{CaC}_{2} \mathrm{O}_{4}+2 \mathrm{NaCl}$. The extremely high solubility of $\mathrm{NaCl}$ compared to $\mathrm{CaOx}$ prevents precipitation of reagents as previously shown ex situ [3]. CaOx formed on the upper window of the LF (Figure $1 \mathrm{C}$ and D). The octahedral morphology of the CaOx is indicative of $\mathrm{CaOx}$ in the dihydrate hydration state (COD), in contrast to previous ex situ synthesis of the more stable $\mathrm{CaOx}$ monohydrate (COM) synthesized via chemical reaction in bulk solution $[3,4]$.

The observed COD on the SiN window may be due to preferential binding of COD from crystals nucleated within the bulk solution while other crystal structures do not bind and flow out of the liquid holder. COD has a higher zeta potential which may cause the $\mathrm{CaOx}$ to adhere to the hydrophilic SiN to increase the stability of the mineral as compared to the bulk solution. The $\mathrm{CaOx}$ not adhered to the LF holder may then freely flow through the chip and outlet. In contrast, COD has been previously shown not to bind to epithelial cells as tightly as $\mathrm{COM}$, suggesting that alternate $\mathrm{CaOx}$ crystal structures are preferentially excreted [4]. Alternatively, $\mathrm{CaOx}$ ions that attach to the $\mathrm{SiN}$ window and mineralize by classical crystal growth may preferentially form COD due to the occlusion of the lower-energy crystal planes necessary to form COM. In Figure $1 \mathrm{C}$ and D, several different sizes of the octahedral COD morphology are shown. The COD crystals range from 50nm to 500nm across; the former being smaller than Abbe's limit. This nanoscale mineralization demonstrates the necessity of high-resolution imaging to study the in situ nucleation, growth, and adhesion of $\mathrm{CaOx}[5]$. 


\section{References:}

[1] M Nielsen, S Aloni, J De Yoreo, Science 345 (2014), p. 1158.

[2] E Hyams, B Matlaga, Translation Andrology and Urology 3 (2014), p. 278.

[3] D Banner et al, Microscopy and Microanalysis 23 (2017), p. 1208.

[4] V Thongboonkerd, T Semangoen, S Chutipongtanate, Clinca Chimica Acta 367 (2006), p. 120.

[5] The authors gratefully acknowledge Nonwovens Institute award \#16-195, NSF award \#1710049, and NSF CAREER award \#1564950. This work made use of instruments in the Electron Microscopy Service (Research Resources Center, UIC).
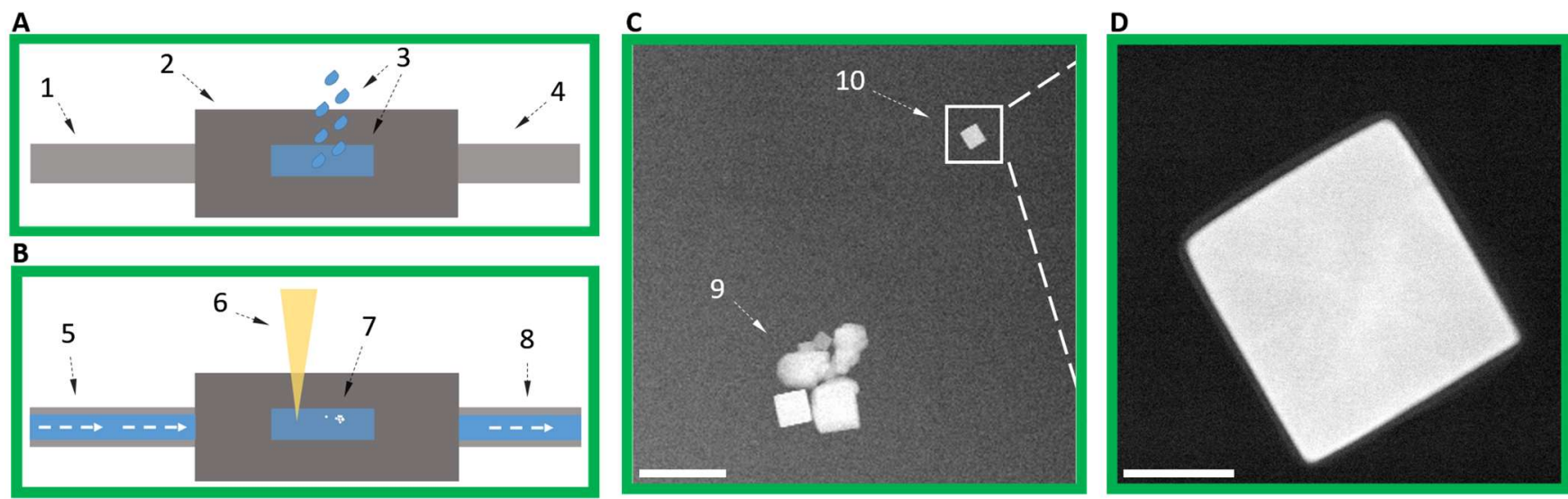

Figure 1. A and $\mathrm{B}$ show diagrams of the liquid flow holder. In $\mathrm{A}$, the $\mathrm{CaCl}_{2}$ solution is added to the $\mathrm{SiN}$ lower window before enclosure by the upper SiN window. A1 indicates the inlet tubing of the chip, A2 the metal frame of the chip, in $\mathrm{A} 3$ the $\mathrm{CaCl}_{2}$ is added to the silicone window, and in $\mathrm{A} 4$ the outlet tube is indicated. In $\mathrm{B}, \mathrm{NaOx}$ flows into the chip through the inlet (B5), the electron beam is used to examine the chip (B6), the material forms on the surface of the SiN window (B7), while the excess fluid and unattached particles exit the chip (B8). $\mathrm{C}$ shows a low magnification image of the $\mathrm{CaOx}$ attached to the surface windows as indicated by B7, labeled C9 and C10, while D shows a high magnification image of the particle indicated as C10. Scale bar in C is $1 \mu \mathrm{m}$, in D the scale bar is $100 \mathrm{~nm}$. 